\title{
O Modelo E-Score de Previsão de Falências para Empresas de Internet
}

\author{
Orlando Mansur Pereira \\ Walter Lee Ness Jr.
}

\begin{abstract}
Resumo
Este estudo propõe um modelo de regressão logística que estime a probabilidade do evento de falência em empresas de Internet, isto é, as que sejam dependentes do e-business. Foram selecionadas 61 empresas norte-americanas e divididas em duas amostras: 25 empresas falidas, entre 1999 e 2001, e 36 não-falidas, no mesmo período. Uma amostra de treinamento foi aleatoriamente selecionada para a determinação das variáveis e dos parâmetros do modelo. A amostra de teste confirmou a alta acurácia do modelo, classificando corretamente 95,1\% da amostra de 61 empresas, utilizando as demonstrações financeiras anuais do ano anterior à falência; e 88,1\%, para demonstrações de 2 anos antes do mesmo evento. Portanto, o Modelo e-Score demonstra ser bastante significante utilizando apenas três variáveis, de 63 analisadas: (i) INT/TL: Interest Expense (Income) / Total Liabilities; (ii) R\&D/EMP: Research \& Development Expenses / No de Empregados; e (iii) OCF/CL: Operating Cash Flow / Current Liabilities. As variáveis (i) e (iii) são obtidas nas demonstrações convencionais, enquanto (ii) é inovadora, indicando que uma empresa de Internet que investe em R\&D por empregado possui maiores chances de sobreviver. Já as que possuem Resultados Financeiros negativos altos e Fluxos de Caixa Operacionais baixos possuem menos chances de sobrevivência.
\end{abstract}

Palavras-chave: modelos de previsão de falências; internet; e-business; regressão logística; modelagem estatística.

\begin{abstract}
This study proposes a binomial logit model to estimate the probability of an event of bankruptcy for Internet companies, i.e. companies dependent on e-business. Sixty-one American companies were selected and divided into two samples: 25, which had filed for petition under the US Bankruptcy Code, between 1999 and 2001, and 36, which had not, for the same period. A training sample was randomly selected to determine the model variables and parameters and a holdout sample has confirmed its high accuracy by correctly classifying $95.1 \%$ of the total sample of 61 companies, based on annual financial statements as of one year before the bankruptcy's occurrence; and $88.1 \%$, as of two years prior to the same event. Then, the e-Score Model reveals to be very significant by using only three variables, of 63 first analyzed: (i) INT/TL: Interest Expense (Income) to Total Liabilities; (ii) R\&D/EMP: Research \& Development Expenses to Number of Employees; e (iii) OCF/CL: Operating Cash Flow to Current Liabilities. While (i) and (iii) can be obtained from conventional financial statements, (ii) is innovative, indicating the company which invests in R\&D on a per employee basis has a greater chance of survival. In contrast, companies presenting relatively high Net Interest Expenses and low Operating Cash Flow have less chance for survival.
\end{abstract}

Key words: bankruptcy prediction models; internet; e-business; logit regression; statistical modeling. 


\section{INTRODUÇÃO}

A Nova Economia, pautada em características como velocidade nas mudanças organizacionais, priorização dos recursos humanos como fonte de riqueza de uma empresa e, principalmente, fechamento de negócios por intermédio da Internet, sofreu impactos, que fizeram soar um alerta nos investidores e credores. Estes impactos traduziram-se, especialmente, nas falências de várias empresas que se propuseram a operar no comércio eletrônico, sendo ele B2B (Business to Business) ou B2C (Business to Consumer). Portanto investidores e credores perderam seu capital aplicado em muitos desses empreendimentos, ditos eletrônicos.

Provavelmente tais investidores teriam apreciado possuir um modo de prever, com alguma antecedência, a situação financeira dessas empresas. Um método usualmente utilizado para este fim é a análise de índices extraídos das demonstrações financeiras ou análise de balanços que, entre outros objetivos, mede solvência, rentabilidade, grau de alavancagem e prazos médios de giro de ativos de uma empresa. Entretanto esses índices foram elaborados com o objetivo de analisar as chamadas empresas tradicionais, que diferem das empresas de Internet, as quais procuram valorizar Ativos Intangíveis, como a marca e o capital humano, por exemplo.

Portanto, se faz necessário realizar uma análise das características financeiras desse novo setor da economia, ou seja, conhecer quais variáveis são ou não significantes para que uma empresa desse setor possa honrar seus compromissos, mantendo uma situação financeira equilibrada. A identificação dessas variáveis permitiria avaliar a situação financeira de uma empresa por intermédio de modelos estatísticos que pudessem estimar a probabilidade de uma empresa falir ou não. Deste modo, o objetivo deste estudo é elaborar um modelo estatístico que estime, por intermédio de um score, denominado $\boldsymbol{e}$-Score, a probabilidade das empresas consideradas de Internet de falir ou entrar em concordata, a partir das variáveis identificadas.

\section{Referencial Teórico}

\section{A Regressão Logística}

A Regressão Logística é uma técnica de modelagem estatística que possui 
como objetivo discriminar dois grupos de observações dentro de uma amostra. Difere da regressão linear, pois seu modelo gera uma variável dependente dicotômica ou binária (Hosmer e Lemeshow, 1989). Desta forma, a variável dependente assume apenas dois valores, ou melhor, está contida em um intervalo finito de valores, entre 0 e 1 . Como o objetivo deste estudo é a análise de um estado binário da situação financeira de uma empresa de Internet, ou seja, se esta empresa está falida ou concordatária, ou não, parece bem provável que essa modelagem seja adequada para os fins desta pesquisa, o que será comprovado adiante. O modelo genérico de regressão logística pode ser expresso da seguinte forma:

$$
\pi(x)=\frac{e^{\beta_{0}+\beta_{1} x_{1}+\beta_{2} x_{2}+\cdots+\beta_{i} x_{i}}}{1+e^{\beta_{0}+\beta_{1} x_{1}+\beta_{2} x_{2}+\cdots+\beta_{i} x_{i}}}
$$

onde: $\pi(x)=E(Y \mid x)$, ou seja, o valor esperado de Y para uma determinada observação $\mathrm{x}$.

Fazendo uma transformação da equação acima, em termos de $\pi(x)$, tem-se que:

$$
g(x)=\ln \left[\frac{\pi(x)}{1-\pi(x)}\right]=\beta_{0}+\beta_{1} x_{1}+\beta_{2} x_{2}+\ldots+\beta_{i} x_{i}
$$

Simplificando, o modelo também pode ser representado da seguinte forma:

$$
\pi(x)=\frac{1}{1+e^{-g(x)}}
$$

onde $g(x)$ representa uma regressão linear e, portanto, apresenta as particularidades dessa regressão, principalmente no que tange à sua propriedade de continuidade da variável dependente. Pode-se perceber, por meio desse modelo, por que a variável dependente, $\pi(x)$, apresenta valores maiores ou iguais a zero e menores de 1 ou iguais a 1 , já que o denominador do modelo nunca será negativo, pois o resultado de $e^{-g(x)}$ sempre será positivo.

Outra característica importante desse modelo é que $\pi(x)$ representa, dada uma observação $x$, a probabilidade de determinado evento Y ocorrer, isto é, de que Y seja igual a 1. Portanto, determinando-se as variáveis da função $g(x)$ para uma determinada observação $x$, temos a probabilidade do estado qualitativo a ser previsto que, neste estudo, significa a ocorrência ou não de falência.

A distribuição da variável dependente do modelo segue uma distribuição binomial, 
com probabilidade dada pela média esperada $\pi(x)$ (Hosmer e Lemeshow, 1989). A curva possui formato de $S$, já que quanto maiores ou menores os valores das variáveis independentes, a variação no valor esperado é muito pequena, ficando o valor esperado próximo de 0 ou 1.

Pode-se, então, fazer uma análise dos resultados obtidos através de $g(x)$ e do valor esperado de $\pi(x)$, isto é, a probabilidade de ocorrência do evento (falência ou não). Quando $g(x)$ tende a $-\infty$, o resultado de $e^{-g(x)}$ tende a $+\infty$, fazendo com que o denominador do modelo se torne grande o suficiente para que $\pi(x)$ tenda a zero. Quando $g(x)$ tende a $+\infty$, o denominador do modelo fica muito próximo de 1 , fazendo com que $\pi(x)$ tenda a 1 .

O modelo de regressão logística utiliza, para determinar os coeficientes das variáveis da função $g(x)$, o método da máxima verossimilhança, o qual estima valores para os parâmetros desta função ( $\beta$ 's).. para que estes maximizem a probabilidade de se obterem os dados observados (Hosmer e Lemeshow, 1989). A função de máxima verossimilhança, também chamada de Deviance, é o resultado do logaritmo neperiano da razão entre a máxima verossimilhança do modelo atual e a máxima verossimilhança do modelo sem nenhuma variável, multiplicado por 2 e, na regressão logística, possui papel semelhante àquele que a Soma dos Erros ao Quadrado desempenha na Regressão Linear (Hosmer e Lemeshow, 1989, p. 13-14).

Através da diferença da Deviance de um modelo sem uma variável e a Deviance do modelo com uma variável é encontrada a estatística $\mathbf{G}$, a qual atesta significância ao modelo e apresenta uma distribuição $\chi^{2}$ com $i$ graus de liberdade, onde $i$ representa o número de variáveis independentes encontradas para o modelo (Hosmer e Lemeshow, 1989, p. 14-16).

O $\mathbf{R}^{2}$ de uma regressão logística demonstra a acurácia do modelo em predizer o valor observado e duas medidas podem ser utilizadas para tal objetivo, o $\mathrm{R}^{2}$ de Cox e Snell e o $\mathrm{R}^{2}$ de Nagelkerke, sendo este último o mais adequado para medir um modelo, já que o $\mathrm{R}^{2}$ de Cox e Snell apresenta como valor máximo 0,75, enquanto o de Nagelkerke fica situado entre 0 e 1 (Minussi, 2001).

A significância de cada variável para o modelo é verificada pelo teste estatístico Wald, representado pela fórmula $W=\left(\beta_{i} / S E_{\beta_{i}}\right)^{2}$, onde $S E$ representa o erro-padrão para o coeficiente estimado, e que possui aproximadamente uma distribuição $\chi^{2}$ com 1 grau de liberdade (Sincich, 1996).

A influência das variáveis independentes no modelo de regressão logística 
pode ser medida pelo antilog do coeficiente $\beta_{i}\left(e^{\beta_{i}}\right)$ de cada variável independente $\left(x_{i}\right)$. O valor de $e^{\beta_{i}}$ representa a mudança nas chances de se observar o evento a ser previsto pelo modelo $(y=1)$, quando a variável independente associada a este coeficiente é elevada em uma unidade, mantendo-se todas as outras variáveis constantes (Sincich, 1996). Já a expressão $e^{\beta_{i}}-1$ indica um percentual de chances de se observar o evento (Sincich, 1996). Desta forma, um percentual negativo demonstra quanto as chances do evento a ser previsto diminuem com o aumento de uma unidade na variável independente associada a este coeficiente, mantendo-se as outras variáveis independentes fixas. A conclusão inversa pode ser tirada, quando o percentual encontrado é positivo.

\section{Estudos Publicados}

Modelos de previsão de dificuldades financeiras de empresas já foram amplamente discutidos no Brasil e no exterior, indicando o interesse do meio acadêmico em tal assunto, já que este possui grande importância para as instituições financeiras e empresas em geral.

Nos estudos pesquisados, três metodologias diferentes foram utilizadas: Análise Discriminante (Altman, 1968; Deakin, 1972; Sinkey, 1975; Altman, Baydia e Ribeiro Dias, 1977; Matias, 1978; Kanitz, 1978; Marques, 1980; Bragança e Bragança, 1985; Martins e Samanéz, 1987; Kasznar, 1986; Nunes, 1988 e Santos, 1996); Regressão Logística (Ohlson, 1980; Matias e Siqueira, 1996; Janot, 1999; Fichman, 2001 e Minussi, Damacena e Ness, 2002); e Redes Neurais Artificiais (Cabrera, 1998).

Em geral, os estudos analisam empresas de setores industriais da economia (Altman, 1968; Altman, Baydia e Ribeiro Dias, 1977; Matias, 1978; Ohlson, 1980; Santos, 1996 e Minussi, Damacena e Ness, 2002), destacando-se os estudos destinados às instituições financeiras (Sinkey, 1975; Martins e Samanéz, 1987; Matias e Siqueira, 1996; Cabrera, 1998; Janot, 1999 e Fichman, 2001).

A grande contribuição gerada por todos estes estudos é a identificação de variáveis ou índices que sejam significativos para discriminar empresas que estejam em boa ou má situação financeira. A Tabela 1 apresenta os índices identificados em alguns dos estudos citados. 


\section{Tabela 1: Índices ou Variáveis de Alguns dos Estudos Citados}

\begin{tabular}{|l|l|}
\hline \multicolumn{1}{|c|}{ Autor } & \multicolumn{1}{c|}{ Índices } \\
\hline Altman (1968) & $\begin{array}{l}\text { Capital Circulante Líquido / Ativos Totais; Lucros Retidos / Ativos Totais; Lucros Antes de } \\
\text { Juros e Imposto de Renda / Ativos Totais; Valor de Mercado do Patrimônio Líquido / Valor } \\
\text { Contábil do Passivo Total e Vendas / Ativos Totais. }\end{array}$ \\
\hline Matias (1978) & $\begin{array}{l}\text { Patrimônio Líquido / Ativo Total; (Financiamentos + Empréstimos Bancários) / Ativo } \\
\text { Circulante; Fornecedores / Ativo Total; Ativo Circulante / Passivo Circulante; Lucro } \\
\text { Operacional / Lucro Bruto e Disponível / Ativo Total }\end{array}$ \\
\hline Kanitz (1978) & $\begin{array}{l}\text { (Ativo Circulante - Estoques) / Passivo Circulante; (Ativo Circulante + Ativo Realizável a } \\
\text { Longo Prazo) / Passivo Exigível a Longo Prazo; Lucro Líquido / Patrimônio Líquido; } \\
\text { Passivo Total / Patrimônio Líquido e Ativo Circulante / Passivo Circulante. }\end{array}$ \\
\hline $\begin{array}{l}\text { Bragança e } \\
\text { Bragança (1985) }\end{array}$ & $\begin{array}{l}\text { Passivo Total / Patrimônio Líquido; Fundo das Operações / Passivo Total; Receitas Líquidas } \\
\text { Ativo Total; Período Médio de Estoques e (Fundo das Operações + Despesas Financeiras) / }\end{array}$ \\
\hline Kasznar (1986) & $\begin{array}{l}\text { Capital de Giro / Ativo Total; Capital Não-Exigível Total Líquido / Ativo Total; Lucro } \\
\text { Antes do Imposto de Renda e Juros / Ativo Total; Valor de Mercado do Capital Social / } \\
\text { Passivo Total e Vendas Líquidas / Ativo Total. }\end{array}$ \\
\hline Nunes (1988) & $\begin{array}{l}\text { Lucro Operacional / Ativo Total; Fluxo de Caixa Operacional / Total das Origens de } \\
\text { Recursos; (Patrimônio Líquido - Ativo Permanente) / Ativo Total; Patrimônio Líquido / } \\
\text { Ativo Total e Lucro Operacional / Lucro Bruto. }\end{array}$ \\
\hline $\begin{array}{l}\text { Mantos (1996) } \\
\text { Damassivo Circulante / Ativo Total; Despesas Financeiras / Passivo Total; Participação de } \\
\text { Ness (2002) } \\
\text { Financiamentos de Instituições de Crédito no Exigível Total da Empresa / Participação de } \\
\text { Financiamentos de Instituições de Crédito no Exigível Total do Setor e Liquidez Geral da } \\
\text { Empresa / Liquidez Geral do Setor. }\end{array}$ \\
\hline $\begin{array}{l}\text { (Passivo Circulante / Patrimônio Líquido) / Mediana do Setor; Investimento Operacional em } \\
\text { Giro / Vendas Líquidas; Tesouraria / Vendas Líquidas; Estoques / Custo das Mercadorias } \\
\text { Vendidas; Obrigações Tributárias e Previdenciárias / Venda Média Mensal e Dívidas } \\
\text { Bancárias de Curto Prazo / Ativo Circulante. }\end{array}$ \\
\hline
\end{tabular}

\section{Metodologia}

\section{Universo e Amostras}

O universo considerado para este estudo é o conjunto de empresas de Internet, ou seja, aquelas que têm como fontes de receita alguma operação vinculada a esta, podendo ser fornecedoras de equipamentos, informações ou realizadoras de negócios por meio da rede. Foram utilizadas empresas sediadas nos EUA e que possuíam ações listadas em bolsas de valores norte-americanas ou em mercados de balcão, pois o mercado brasileiro carece de empresas de Internet de capital aberto; assim, o acesso às informações para um estudo nacional sobre este assunto é impraticável.

Neste tipo de estudo devem ser consideradas duas amostras: uma, de empresas que não faliram nem entraram em concordata; e outra, de empresas que já efetuaram estes procedimentos. No que concerne às empresas que não faliram, 
foi utilizada como primeira indicação de quais eram essas empresas, aquelas listadas no índice Inter@ctive Week Internet Index da AMEX (American Stock Exchange) em maio de 2002. A partir deste índice, foram analisadas todas as empresas que o compõem, sendo estudados os seus segmentos de atuação por meio da Internet e seus competidores, os quais não faziam parte do índice. Desta forma, foi realizada uma amostragem por conveniência, sendo selecionadas as empresas que mais se adequavam como empresas pertencentes ao segmento de Internet.

A amostragem por conveniência se justifica no fato de que este estudo pretende utilizar, em suas amostras, empresas que não sobreviveriam sem a existência da Internet. De fato, uma empresa que produz softwares B2B para Portais de Internet, sem a existência desta, perderia seu único cliente. Entretanto empresas que prestam consultorias em vários segmentos, sendo um deles o de Internet, não deveria ser considerada como de Internet. Afinal, se esta não existisse, a empresa sobreviveria, prestando consultoria nos outros segmentos.

Desta forma, foram selecionadas 36 empresas não-falidas (a partir deste momento, serão assim chamadas estas empresas) com Ativos Totais variando de US\$900 mil a US\$32,9 bilhões, permitindo que a amostra seja a mais diversificada possível em relação ao tamanho da empresa e também ao número de empregados; este variando de 10 a 34.000 empregados.

Essa diversificação não acarreta viés para o estudo, pois o que se busca são variáveis que, por meio de razões, possam explicar determinado comportamento da empresa; neste caso, falir ou não. Portanto, a empresa, sendo grande ou pequena, deveria manter essas razões proporcionais ao seu porte, para que consiga atingir o comportamento de não-falência. As empresas não-falidas selecionadas foram: @pos.com Inc.; EarthLink, Inc.; Accrue Software, Inc.; FreeMarkets, Inc.; Actuate Corporation; Global Web, Inc.; Akamai Technologies, Inc.; Homestore, Inc.; American Software, Inc.; Hotels.com; Amazon.com, Inc.; Internet Security Systems, Inc.; Activeworlds Corp.; Macromedia, Inc.; BEA Systems, Inc.; Network Associates, Inc.; Check Point Software Technologies Ltd.; Overture Services, Inc.; CheckFree Corporation; Pacific WebWorks, Inc.; Commerce One, Inc.; Priceline.com Inc.; Cisco Systems, Inc.; RealNetworks, Inc.; DoubleClick Inc.; RSA Security Inc.; Digital Insight Corp.; SeeBeyond Technology, Corp.; eBay, Inc.; Siebel Systems, Inc.; eCollege.com; WebMD Corporation; eAutoclaims.com, Inc.; VeriSign, Inc.; E*TRADE Group, Inc. e Yahoo! Inc.

No caso de empresas que faliram ou entraram em concordata, foram selecionadas aquelas que já preencheram algum pedido de falência nas Cortes de Falências norte-americanas. Conforme as informações do tipo de negócio, 
seguindo o conceito de empresas de Internet e os mesmos critérios citados nos parágrafos anteriores, foram selecionadas 25 empresas falidas, com Ativos Totais variando de US $\$ 7,3$ milhões a US $\$ 4,5$ bilhões e número de empregados variando de 28 a 4.290. As empresas falidas, utilizadas neste estudo, foram AHT Corporation; MerchantOnline.com, Inc.; audiohighway.com; Motient Corporation; Ardent Communications, Inc.; MyTurn.com, Inc.; AppliedTheory Corporation; Osage Systems Group, Inc.; Beyond.com Corporation; PSINet Inc.; Caliber Learning Network, Inc.; Quintus Corporation ; CyberCash, Inc.; Scient, Inc. ; drkoop.com, Inc.; SoftLock.com, Inc.; ESNI, Inc.; Startec Global Communications Corp.; Exodus Communications Inc.; U.S. Interactive, Inc.; HealthCentral.com; World Access, Inc.; iBEAM Broadcasting Corp.; Xpedior, Inc. e MicroAge, Inc..

Na amostra de empresas falidas já não se consegue observar uma diversificação tão grande no tamanho e número de empregados; porém, da mesma forma, isto não acarreta viés para a amostra, pois o comportamento a ser testado já está comprovado. Estas empresas, efetivamente, já entraram em processo de falência ou concordata nas cortes norte-americanas.

\section{Coleta de Dados}

A coleta de dados foi realizada por intermédio do software Edgar Online, o qual dá acesso a todos os relatórios obrigatórios que as empresas norte-americanas devem preencher para atender aos requisitos da SEC (Securities and Exchange Commission). $\mathrm{O}$ acesso às informações é efetuado por meio da Internet pelo site www.edgar-online.com e foram coletadas as demonstrações financeiras das empresas, ou seja, Balanço Patrimonial, Demonstração de Resultados e Fluxo de Caixa.

Os dados para a utilização das variáveis foram extraídos dos formulários 10-K e complementares de cada empresa, os quais representam os relatórios anuais aos acionistas, referentes aos anos de 2000, 1999, 1998. Entretanto, alguns dados necessários para as variáveis, como o número de empregados, e outras informações para a seleção da amostra, como o escopo do negócio de cada empresa, foram coletados da empresa Multex.com. Esta empresa, através de seu site Multex Investor (www.multexinvestor.com), oferece várias informações sobre empresas, tais como: preços históricos de ações, análise de índices de balanços, perfil de negócios, informações sobre insiders, dentre outras.

\section{Tratamento de Dados}

Os dados foram tratados com auxílio do software estatístico SPSS 10.0. A metodologia utilizada foi a seguinte: verificar, mediante pesquisa bibliográfica, 
quais índices são mais corriqueiramente mencionados; calcular estes índices para as empresas de Internet; testar a significância destes índices na discriminação das duas amostras de empresas falidas e não-falidas; elaborar novas variáveis e calculá-las; de todas as variáveis, identificar quais possuem maior significância para o modelo a ser proposto e elaborá-lo e testar a sua acurácia por meio do percentual de classificação correta e de outros testes estatísticos costumeiramente aplicados à Regressão Logística.

\section{Variáveis Estudadas}

Uma etapa crucial deste estudo é a determinação das variáveis mais significantes para o ambiente de negócios de Internet, já que nenhum dos estudos anteriores citados utilizou as empresas de Internet em suas amostras. Obviamente, o que para uma empresa industrial é importante, para uma empresa prestadora de serviços, por exemplo, pode não ser. Desta forma, o modelo a ser gerado por este estudo tem como objetivo ser aplicado apenas ao setor de empresas de Internet.

As variáveis foram elaboradas, utilizando grupamentos contábeis do Balanço Patrimonial (Contas a Pagar, Ativo Imobilizado etc.), da Demonstração de Resultados (Receita Bruta, EBITDA etc.) ou da Demonstração de Fluxo de Caixa (Fluxo Operacional, de Investimentos etc.). Neste sentido, foram utilizadas demonstrações financeiras de um ano anterior ao evento. No caso de empresas falidas, se torna fácil estabelecer este ano, pois a data do pedido de falência é conhecida. No caso de empresas não-falidas, para que não houvesse nenhuma influência sazonal do comportamento do setor de negócios, as demonstrações financeiras utilizadas obedeceram à mesma distribuição de freqüência das demonstrações das empresas falidas.

Seguindo esses parâmetros, foram testadas as 63 variáveis que se encontram relacionadas no Anexo 1. Primeiramente, por meio do teste de Levene, foi testada a igualdade das variâncias em nível de significância de 5\%; após este passo, foi utilizado um Teste t para a igualdade das médias, também em nível de significância de 5\%. Desta forma, 26 variáveis demonstraram ser significantes (hipótese de igualdade das médias rejeitada), que estão destacadas no Anexo 1.

Dessas 26 variáveis foram selecionadas 18 que possuem maior significância e foi testada a normalidade da distribuição destas para a amostra com 61 empresas: 36 empresas não-falidas e 25 empresas falidas. Foi utilizado o Teste de Kolmogorov-Smirnov e este indicou, em nível de significância de 5\%, que apenas três variáveis (NWC/TA A1, CASH/TA, AP/TA e GP/TA) possuíam uma distribuição normal. Desta forma, foi utilizado, ainda, o índice de Spearman, o qual é uma versão não-paramétrica da correlação de Pearson, para medir a 
correlação entre essas variáveis, em nível de significância de 5\%, já que não é desejável que ocorra o fenômeno da multicolinearidade. Foram, então, retiradas as variáveis que mais possuíam correlações, restando para serem utilizadas na geração do modelo as seguintes variáveis.

\section{. PPE / TA: Property, Plant \& Equipment / Total Assets}

Esta variável tenta medir o grau de investimentos em Ativos Imobilizados de uma empresa, que no caso das de Internet é muito importante, por se tratar de empresas de tecnologia. As empresas não-falidas demonstraram investir menos em Ativos Imobilizados, apresentando uma média de apenas 0,08 do Ativo Total. As empresas falidas apresentaram uma média maior, de 0,22, demonstrando que, em empresas de Internet, imobilizar capital em Ativos pode ser maléfico à empresa. É importante salientar que foram utilizados os valores líquidos, já descontados da depreciação, a qual pode estar influenciando estas médias, pois empresas mais antigas provavelmente terão seus Ativos Imobilizados Líquidos menores que o de empresas mais novas. Esta variável apresentou correlações significantes com (CA+LTA)/TL, D/CA, D/TL, GP/REV, R\&D/EMP 1000, OCF/BA, OCF/EMP 1000, OCF/SHREQ e EBITDA/EMP 1000.

\section{. INT / TL: Interest Expenses (Income) / Total Liabilities}

Esta variável apresenta questões bem interessantes, pois como está sendo utilizado o Resultado Financeiro Líquido, seu valor pode apresentar sinal positivo ou negativo. Ao apresentar sinal positivo, esta variável é uma aproximação do custo de capital de terceiros de uma empresa. O sinal negativo indica que uma empresa possui mais receitas financeiras do que despesas financeiras, ou seja, está operando bem o seu caixa em relação aos seus empréstimos ou, ainda, está sendo totalmente financiada pelos acionistas. As empresas não-falidas apresentaram um média de -0,04, indicando que seu resultado financeiro líquido representa algo em torno de $4 \%$ do seu Passivo Total. Já as empresas falidas apresentaram uma média de 0,02, indicando que pagam, aproximadamente, 2\% de juros sobre o seu Passivo Total. Esta variável apresentou correlações significantes com CA/CL, (CA+LTA)/TL, NWC/TAA1, CASH/TA, CASH/CL, D/CA, D/TL, AP/TA, GP/REV e OCF/SHREQ.

\section{. GP / TA: Gross Profit / Total Assets}

Esta variável é uma medida de rentabilidade e permite observar o retorno operacional que os Ativos de uma empresa geram para os acionistas. Portanto, quanto maior este índice, melhor para uma empresa. Espera-se, ainda, que seja sempre maior que zero, pois se uma empresa apresenta Prejuízo Bruto, isto 
significa que as receitas são insuficientes até para cobrir os seus custos, sem mencionar, ainda, suas despesas administrativas, de vendas ou gerais. Os resultados encontrados indicaram o grupo de empresas não-falidas com uma média para este índice de rentabilidade de 0,31; e o de empresas falidas com uma média mais baixa, de 0,10. Este índice apresentou correlações significantes com NWC/TA A1, AP/TA, GP/REV, OCF/CL, OCF/BA, OCF/EMP 1000, OCF/SHREQ e EBITDA/EMP 1000.

\section{. R\&D / EMP 1000: Research \& Development Expenses / $\mathrm{N}^{\circ} \mathrm{de}$ Empregados}

Esta é uma nova variável proposta por este estudo. O objetivo dela é medir quanto uma empresa investe em pesquisa e desenvolvimento (em US\$ mil) por funcionário. A justificativa da tentativa de incluir esta variável consiste no fato de que empresas de tecnologia, muitas vezes, têm como ponto forte o capital humano, isto é, a competência e capacidade de seus funcionários. Foi observado que as empresas de Internet possuíam baixos valores contabilizados no grupo de Ativos Imobilizados, em comparação com os seus Ativos Totais. Em compensação, o índice para o Ativo Permanente demonstrou ser mais elevado, indicando que essas empresas investiram muito em projetos e, provavelmente, no desenvolvimento e treinamento de seus funcionários. Poder-se-ia afirmar, ainda, que empresas que demonstram grande importância e são dependentes do capital humano, poderiam medir o seu tamanho pelo número de funcionários e não pelo total de Ativos. Desta forma, empresas que investissem mais em Pesquisa e Desenvolvimento por funcionário teriam mais chances de sucesso. Isto ficou efetivamente demonstrado pelas médias apresentadas pelos dois grupos. O grupo de empresas não-falidas apresentou uma média de US\$30,78 mil por funcionário, enquanto o grupo de empresas falidas, apenas US\$ 10,49 mil. Esta variável apresentou correlações significantes com (CA+LTA)/TL, PPE/TA, CASH/TA, CASH/CL, D/CA, D/TL, AP/TA, GP/REV e FRCF/TA.

\section{. OCF / CL: Operating Cash Flow / Current Liabilities}

Esta também é variável nova, proposta por este estudo. A diferença desta para a Liquidez Imediata (Caixa / Passivo Circulante) é que na Liquidez Imediata não importa a origem do capital utilizado para cobrir o Passivo Circulante, porquanto, como considera o saldo de Caixa, ali podem estar recursos de terceiros (empréstimos) ou de acionistas (aportes de capital). Esta nova variável mede se uma empresa possui condições de pagar seus Passivos Circulantes com a geração própria de caixa. Isto é muito importante, pois demonstra aos acionistas que aquela empresa irá conseguir manter-se sozinha, sem a necessidade de chamá-los a aportar capital ou de tomar empréstimos para bancar o seu Capital Circulante; 
portanto valores positivos e acima de 1 são esperados para esta variável. Quando ocorrem valores negativos, estes indicam que o Passivo Circulante é coberto totalmente por aportes de capital próprio ou financiamentos. O grupo de empresas não-falidas apresentou uma média de -0,17 e o grupo de empresas falidas uma média de -1,74. Esta variável apresentou correlações significantes com AP/TA, GP/TA, GP/REV, OCF/BA, OCF/EMP 1000, OCF/SHREQ e EBITDA/EMP 1000 .

\section{. OCF / EMP 1000: Operating Cash Flow / No de Empregados}

Esta variável tem como objetivo medir quanto de Fluxo de Caixa Operacional cada funcionário de uma empresa gera em US\$ mil. Esta é uma medida inovadora, que neste estudo demonstrou sua eficácia na discriminação de empresas falidas e não-falidas de Internet. A média observada para o grupo de empresas nãofalidas foi de US\$1,32 mil por funcionário. No caso de empresas falidas, a média apresentada foi muito baixa, apontando para o valor de US\$ -163,99 mil por funcionário. Apresentou correlações significantes com CA/CL, (CA+LTA)/TL, PPE/TA, NWC/TA A1, D/CA, AP/TA, GP/TA, GP/REV, OCF/CL, OCF/BA, FRCF/TA, OCF/SHREQ e EBITDA/EMP 1000.

\section{. CASH / TA: Cash / Total Assets}

Este índice é uma tentativa de perceber o quanto uma empresa é geradora de caixa, isto é, o quanto da rentabilidade dos seus Ativos uma empresa acumula em seu caixa e não investe em outros projetos. Pode indicar, ainda, que uma empresa deseja manter uma margem de segurança alta, mantendo elevado seu caixa para possíveis problemas financeiros futuros. O que foi observado é que as empresas de Internet não-falidas possuíam este índice um pouco mais elevado, com média em 0,43, contra 0,27 das empresas falidas, indicando que, com as sucessivas quebras e instabilidade no setor, as empresas não-falidas poderiam estar se preparando para um eventual desequilíbrio financeiro. Esta variável apresentou correlações significantes com CA/CL, (CA+LTA)/TL, NWC/TA A1, CASH/ CL, INT/TL, D/CA, AP/TA, GP/REV, R\&D/EMP 1000 e FRCF/TA.

\section{Resultados}

\section{Comparação com os Modelos Anteriormente Propostos}

As variáveis CA / CL (Matias, 1978; Kanitz, 1978 e Ohlson, 1980), CASH / TA (Matias, 1978), NWC / TA A1 (Altman, 1968 e Kasznar, 1986) e D / CA 
(Matias, 1978) já haviam sido descritas anteriormente por outros autores e se mostraram significantes na discriminação de grupos de empresas falidas e nãofalidas de Internet. Portanto, de dezoito variáveis, consideradas mais significantes para as empresas de Internet, apenas quatro já haviam sido citadas em estudos anteriores, o que denota a diferenciação desse novo setor da Economia. A falta de significância de outras variáveis dos modelos já propostos por outros autores na discriminação de amostras de empresas falidas e não-falidas de outros setores da Economia compromete a utilização desses modelos em predizer a falência de uma empresa de Internet.

\section{O Modelo e-Score de Previsão de Falências para Empresas de Internet}

A amostra total, com 61 empresas, foi dividida em duas subamostras para que o modelo fosse construído com mais rigor e conservadorismo. Estas duas subamostras, chamadas de amostra de treinamento e amostra de teste, foram obtidas de forma aleatória, por intermédio da função Filtrar Casos do SPSS 10.0. A amostra de treinamento foi composta de 38 empresas, 20 empresas nãofalidas e 18 empresas falidas, correspondendo a um percentual de $62 \%$ do total da amostra, e é a que foi utilizada para gerar o Modelo e-Score.

O método utilizado para gerá-lo foi o Forward Stepwise (Wald). Neste método, o objetivo dos algoritmos do software estatístico é maximizar a estatística Wald para cada variável, enquanto aumenta, simultaneamente, a estatística $\mathbf{G}$ para o modelo, fazendo com que seja reduzido o valor da máxima verossimilhança deste. A variável qualitativa dependente (e-Score) foi codificada com os valores 0 e 1 , 0 representando empresas não-falidas e 1 as empresas falidas. $\mathrm{O}$ valor de cutoff utilizado foi de 0,5; portanto este é o Modelo e-Score:

$$
\text { e - Score }=\frac{e^{0,517+47,256 X_{1}-0,213 X_{2}-3,443 X_{3}}}{1+e^{0,517+47,256 X_{1}-0,213 X_{2}-3,443 X_{3}}}, \text { onde: }
$$

$X_{1}=\mathrm{INT} / \mathrm{TL}$ : Interest Expense (Income) / Total Liabilities;

$X_{2}=\mathrm{R} \& \mathrm{D} / \mathrm{EMP}$ 1000: Research \& Development Expenses / $\mathrm{N}^{\mathrm{o}}$ de Empregados e $X_{3}=\mathrm{OCF} / \mathrm{CL}$ : Operating Cash Flow / Current Liabilities.

\section{Análise da Significância do Modelo}

O Modelo e-Score demonstrou ser bastante significante na previsão de falências para empresas de Internet, assim como apresentou alto poder de predição da 
variável dependente. O teste estatístico $\mathbf{G}$ apresentou valor de 41,508, indicando significância de 0,000, em nível de significância de 5\% e com 3 graus de liberdade. O poder de predição é medido pelo $\mathbf{R}^{2}$ de Nagelkerke, que apresentou um valor bem relevante de 0,887 , demonstrando que $88,7 \%$ das situações financeiras das empresas constantes da amostra podem ser explicadas pelo modelo. O modelo obteve uma Deviance de 11,066, o que também demonstra boa capacidade preditiva, já que esta estatística deve ser baixa (Hosmer e Lemeshow, 1989). Os valores desses testes estatísticos estão sumariados na Tabela 2.

\section{Tabela 2: Análise da Significância do Modelo e-Score}

\begin{tabular}{|ccccc|}
\hline & G & Deviance & $\mathbf{R}^{2}$ Cox \& Snell & R $^{2}$ Nagelkerke \\
\hline Modelo e-Score & 41,508 & 11,066 & 0,665 & 0,887 \\
\hline
\end{tabular}

Hosmer e Lemeshow (1989, p. 140-145) propõem ainda outro teste para comprovar a significância de modelo. Este teste, que os autores chamam de $C$, é baseado no número de classificações corretas das empresas em relação ao número que é esperado pelos decis de cada amostra (falidas e não-falidas) e possui uma distribuição $\chi^{2}$ com 8 graus de liberdade. No Modelo e-Score, indicou um valor de 3,477 com significância de 0,901. Desta forma, não se pode rejeitar fortemente a hipótese nula de que o modelo possui acurácia na classificação das duas amostras, em nível de significância de $1 \%$.

\section{Análise da Significância das Variáveis}

A análise da significância das variáveis foi realizada por meio do teste Wald. Este teste possui uma distribuição $\chi^{2} \operatorname{com} 1$ grau de liberdade e testa a hipótese nula de que pelo menos dois parâmetros de duas das variáveis são significantemente iguais. Seus resultados estão apresentados na Tabela 3, demonstrando que todas as variáveis são significantes, em nível de significância de $5 \%$.

Tabela 3: Análise da Significância das Variáveis do Modelo e-Score

\begin{tabular}{|lcccccc|}
\hline \multicolumn{1}{|c}{ Variável } & $\beta$ & Wald & Sig & $\mathbf{e}^{\beta}$ & Min e $^{\beta}$ & Max e $^{\beta}$ \\
\hline INT / TL & 47,256 & 4,794 & 0,029 & $3,34 \mathrm{E}+20$ & 142,073 & $7,83 \mathrm{E}+38$ \\
R\&D / EMP 1000 & $-0,213$ & 5,246 & 0,022 & 0,808 & 0,673 & 0,970 \\
OCF / CL & $-3,443$ & 5,811 & 0,016 & 0,032 & 0,002 & 0,525 \\
Constante & 0,517 & 0,231 & 0,631 & 1,678 & - & - \\
\hline
\end{tabular}

Os sinais demonstrados pelos coeficientes ( $\beta$ 's) indicam que as variáveis $\mathbf{R} \& \mathbf{D}$ / EMP 1000 e OCF / CL influenciam positivamente a empresa, ou seja, fazem 
com que a variável dependente e-Score se aproxime do zero, isto é, do estado de não-falência. A análise do resultado de $e^{\beta}-1$ informa as chances percentuais da diminuição ou aumento da probabilidade do evento, ou seja a falência ocorrer, mantendo-se as outras variáveis constantes. Entretanto, quando o $\beta$ de uma variável é muito alto, como é o caso da variável INT / TL, a análise é prejudicada, pois o valor calculado de $\mathrm{e}^{\beta}-1$ se apresenta muito alto ou muito baixo, não se podendo chegar a conclusões práticas. Portanto, para a variável R\&D / EMP 1000, a cada US\$ 1 mil de Pesquisa e Desenvolvimento investidos a mais por funcionário, as chances da empresa falir diminuem em 19,2\% (0,808 - $1=$ 0,192). Já para a variável OCF / CL a cada $1 \%$ de aumento na relação Fluxo de Caixa Operacional e Passivo Circulante, as chances de uma empresa de Internet falir diminuem em, aproximadamente, $1 \%((0,032$ - 1) / $100=-0,00968)$.

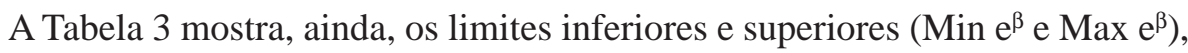
em um intervalo de confiança de $95 \%$, para estas chances. Portanto, o percentual máximo em que uma empresa de Internet conseguiria diminuir as suas chances de falência, se aumentasse em US\$1 mil por funcionário, seria de 32,7\%, ou seja, onde está situado o valor mínimo para o intervalo de confiança (0,673 - 1 = 0,327) da variável R\&D / EMP 1000. Já para a variável OCF / CL, no pior cenário, isto é, no limite superior do intervalo de confiança, o percentual mínimo em que uma empresa de Internet conseguiria diminuir a probabilidade de falência, aumentando em 1\% a relação Fluxo de Caixa Operacional e Passivo Circulante seria de $0,47 \%((0,525-1) / 100=-0,00475)$.

A correlação não-paramétrica (Spearman) foi medida para as três variáveis na amostra de 61 empresas e, conforme demonstra a Tabela 4, não foram identificadas correlações significantes entre as três, em nível de significância de 5\%, indicando a não-ocorrência do fenômeno de multicolinearidade no modelo.

Tabela 4: Correlação entre as Variáveis do Modelo e-Score

\begin{tabular}{|llccc|}
\hline \multicolumn{1}{|c}{ Variável } & & INT / TL & R\&D / EMP 1000 & OCF / CL \\
\hline INT / TL & Coeficiente & 1,000 & $-0,217$ & $-0,102$ \\
& Significância & - & 0,092 & 0,432 \\
\hline \multirow{2}{*}{ R\&D / EMP 1000 } & Coeficiente & $-0,217$ & 1,000 & 0,167 \\
& Significância & $\mathbf{0 , 0 9 2}$ & - & 0,198 \\
\hline OCF / CL & Coeficiente & $-0,102$ & 0,167 & 1,000 \\
& Significância & $\mathbf{0 , 4 3 2}$ & $\mathbf{0 , 1 9 8}$ & - \\
\hline
\end{tabular}

Entretanto este tipo de análise não-paramétrica pode estar mascarando alguma correlação não-linear. Desta forma, foram elaborados gráficos do tipo Scatter Plot para as variáveis, que indicaram a inexistência de algum tipo de padrão de comportamento de correlação entre as variáveis selecionadas para o Modelo e-Score. 
A Tabela 5 apresenta as correlações não-paramétricas entre as variáveis e o eScore gerado pelo modelo e suas respectivas significâncias. Todas as variáveis apresentaram correlações significantes em nível de $1 \%$ e os sinais negativos das variáveis R\&D / EMP 1000 e OCF / CL indicam que estas variáveis se correlacionaram negativamente com o evento de falência.

\section{Tabela 5: Correlações entre as Variáveis do Modelo e-Score e o e-Score Gerado pelo Modelo}

\begin{tabular}{|clccc|}
\hline & & INT / TL & R\&D / EMP 1000 & OCF / CL \\
\hline \multirow{2}{*}{ e-Score } & Correlação & 0,507 & $-0,705$ & $-0,651$ \\
& Significância & 0,000 & 0,000 & 0,000 \\
\hline
\end{tabular}

Portanto, por meio de todas as análises realizadas, as variáveis demonstraram ser bastante significantes para o Modelo e-Score de Previsão de Falências para Empresas de Internet.

\section{Percentual de Acerto na Classificação para Um Ano Antes do Evento}

O percentual de acerto na classificação das empresas é dado pela Tabela de Classificação. Esta tabela deve ser elaborada para cada subamostra designada, ou seja, a de treinamento e a de teste. O Modelo e-Score obteve excelentes resultados na classificação das empresas de Internet na amostra de treinamento, como mostra a Tabela 6.

\section{Tabela 6: Tabela de Classificação do Modelo e-Score na Amostra de Treinamento}

\begin{tabular}{|cccc|}
\hline & \multicolumn{3}{c|}{ Estimadas } \\
\cline { 2 - 4 } Observadas & Não-Falidas & Falidas & Percentual de Acerto \\
\hline Não-Falidas & 19 & 1 & 95,0 \\
Falidas & 0 & 18 & 100,0 \\
\hline & \multicolumn{3}{c}{ Percentual Geral } \\
\hline
\end{tabular}

Na aplicação do modelo, apenas uma empresa não-falida e nenhuma falida foram classificadas erroneamente, atingindo um percentual de acerto de 97,4\%, o que demonstra a sua acurácia. Este percentual pode ser considerado como o percentual máximo a ser atingido pelo Modelo e-Score, já que foi obtido por meio da amostra de treinamento.

Entretanto, é mediante a amostra de teste que o modelo realmente é avaliado, 
já que as empresas que constam desta amostra não estavam incluídas na modelagem das variáveis. A Tabela 7 apresenta os resultados da classificação obtida na amostra de teste.

\section{Tabela 7: Tabela de Classificação do Modelo e-Score na Amostra de Teste}

\begin{tabular}{|cccc|}
\hline & \multicolumn{3}{c|}{ Estimadas } \\
\cline { 2 - 4 } Observadas & Não-Falidas & Falidas & Percentual de Acerto \\
\hline Não-Falidas & 15 & 1 & 93,8 \\
Falidas & 1 & 6 & 85,7 \\
\hline & \multicolumn{3}{c}{ Percentual Geral } \\
\hline
\end{tabular}

Na amostra de teste, o percentual geral de classificação diminui, assumindo o valor de 91,3\%. Portanto o número reduzido de empresas falidas na amostra de teste está impactando negativamente o percentual geral de classificação, pois apenas uma empresa foi classificada erroneamente e isto fez com que o percentual de classificação na subamostra de empresas falidas decaísse quase $15 \%$. No entanto, 91,3\% de classificação correta ainda é um excelente percentual, tendo em vista que os outros critérios, já apresentados neste estudo, apontam a grande significância do Modelo e-Score.

As tabelas de classificação também demonstram os custos de classificação do modelo, ou seja, os erros do Tipo I e II. O erro do Tipo I é considerado a classificação de empresas falidas no grupo de não-falidas e o do Tipo II representa a classificação de empresas não-falidas no grupo de falidas. Por meio da análise destas tabelas de classificação é percebido que o erro do Tipo I para a amostra de treinamento é de $0 \%$ e o do Tipo II de $5 \%$. Na amostra de teste, os erros aumentam o seu percentual, apresentando $14,3 \%$ para o do Tipo I e 6,2\% para o do Tipo II. O Modelo e-Score, quando aplicado na amostra de 61 empresas, indicou o que está representado na Tabela 8.

\section{Tabela 8: Tabela de Classificação do Modelo e-Score na Amostra Total para Um Ano Antes do Evento}

\begin{tabular}{|cccc|}
\hline & \multicolumn{3}{c|}{ Estimadas } \\
\cline { 2 - 3 } Observadas & Não-Falidas & Falidas & Percentual de Acerto \\
\hline Não-Falidas & 34 & 2 & 94,4 \\
Falidas & 1 & 24 & 96,0 \\
\hline & & Percentual Geral & $\mathbf{9 5 , 1}$ \\
\hline
\end{tabular}

Portanto a Tabela 8 indica um acerto global, para o Modelo e-Score, de 95,1\%, com um erro do Tipo I de $4 \%$ e o do Tipo II de 5,6\%. O modelo demonstrou ser 
bastante conservador, pois o erro maior apresentado, o do Tipo II, significa enquadrar uma empresa não-falida como falida. Desta forma, seria muito pior para uma instituição financeira emprestar capital para uma empresa, esperando que ela pague (incorrer no erro Tipo I) do que não emprestar este mesmo capital para outra empresa, achando que ela não irá pagar (incorrer no erro Tipo II).

É necessário que seja efetuada, então, uma análise da sensibilidade do fator de corte da regressão, o qual determina se a empresa possui maior probabilidade de falência ou não. Neste estudo, o percentual de corte utilizado foi o de $50 \%$, ou seja, as empresas de Internet possuem a mesma probabilidade de falir ou não.

Deste modo, foi elaborado um histograma com os e-Scores gerados pelo modelo para as 61 empresas e 31, das 36 empresas não-falidas, apresentaram um eScore entre 0 e 0,1 . Dentre as empresas falidas, 13 das 25 apresentaram um eScore entre 0,9 e 1; 5 entre 0,8 e 0,9; 3 entre 0,7 e 0,8; 2 entre 0,6 e 0,7; e 1 entre 0,5 e 0,6 . Estes comportamentos indicam que, deslocando o ponto de corte para direita, estar-se-ia aumentando o erro do Tipo I, pois mais empresas falidas estariam sendo classificadas como não-falidas, o que é muito temeroso. O Gráfico 1 demonstra uma simulação do ponto de corte e o erro do Tipo I, indicando que para o Modelo e-Score o ideal é manter o ponto de corte em 0,5.

\section{Gráfico 1: Simulação do Aumento do Erro do Tipo I com a Mudança no Ponto de Corte}

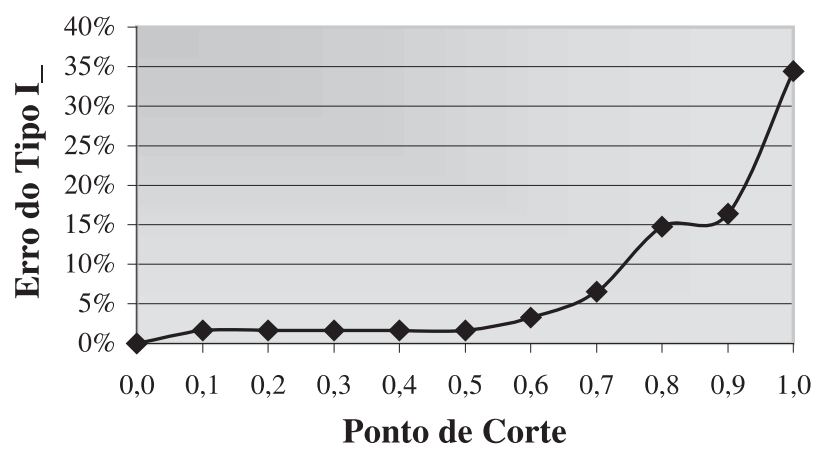

\section{Percentual de Acerto na Classificação para Dois Anos Antes do Evento}

O percentual de classificação indicado pelo Modelo e-Score pode estar sendo influenciado pelo caráter momentâneo das demonstrações financeiras, pois estas retratam determinado comportamento em um período específico da vida de uma empresa. No intuito de testar o comportamento no tempo das variáveis do modelo 
e-Score, este foi aplicado à amostra total, utilizando as demonstrações financeiras de dois anos anteriores ao evento observado: falência ou não-falência. Os percentuais de classificação correta para este período estão representados na Tabela 9.

\section{Tabela 9: Tabela de Classificação do Modelo e-Score na Amostra Total para Dois Anos Antes do Evento}

\begin{tabular}{|cccc|}
\hline & \multicolumn{3}{c|}{ Estimadas } \\
\cline { 2 - 3 } Observadas & Não-Falidas & Falidas & Percentual de Acerto \\
\hline Não-Falidas & 32 & 3 & 91,4 \\
Falidas & 4 & 20 & 83,3 \\
\hline & & Percentual Geral & $\mathbf{8 8 , 1}$ \\
\hline
\end{tabular}

O percentual indicado de 88,1\% sinaliza a eficiência das variáveis apresentadas no modelo, demonstrando que elas podem ser consideradas como boas indicadoras desse novo setor da economia, pois não refletem uma situação momentânea das empresas.

Comparando com a aplicação do modelo para um ano antes do evento observado, foi constatado que 3 empresas falidas tiveram seus e-Scores alterados de 0 para 1 , indicando que a situação financeira dessas empresas se deteriorou bastante em apenas 1 ano. Em relação às empresas de Internet não-falidas, o modelo classifica 3 destas como falidas, para dois anos antes do evento, aumentando em apenas 1 empresa o número de estimativas incorretas, quando comparado à sua aplicação para um ano antes do evento observado.

A alteração do número total de empresas utilizadas na amostra de 61 para um ano e 59 para dois anos antes do evento deveu-se à não disponibilidade de informações referentes ao número de empregados de duas empresas para os períodos necessários, o que inviabilizou o cálculo da variável R\&D / EMP 1000 e do e-Score para estas empresas.

\section{Conclusão}

O mercado financeiro necessita possuir instrumentos que possam, efetivamente, predizer um futuro próximo, para que sejam evitadas grandes perdas de capital, como as que ocorreram com as empresas de Internet. Os estudos anteriores, aqui citados, tiveram sempre esta preocupação, ou seja, fornecer ferramentas ao mercado, para que este possa melhor analisar as suas empresas participantes. 
O Modelo e-Score para Previsão de Falências de Empresas de Internet demonstrou ser bastante eficiente ao estimar a probabilidade de falência ou concordata dessas empresas, atingindo um percentual máximo de 97,4\%. Identificou, ainda, características financeiras importantes das empresas de Internet, que são traduzidas pelas variáveis demonstradas no modelo e que ainda não haviam sido utilizadas por autores em estudos similares prévios.

A variável INT / TL representa qual o resultado financeiro líquido uma empresa obteve em relação ao seu Passivo Total. Se uma empresa apresenta resultado negativo para esta variável, isto significa que seus recursos financeiros estão sendo bem geridos, indicando para uma maior existência de receitas, em relação às despesas financeiras. Quando uma empresa apresenta resultado positivo, isto demonstra que o seu estoque de dívida gera mais encargos financeiros do que suas aplicações geram rendimentos. Portanto, quanto maior esta variável, mais a empresa está sendo “castigada” financeiramente, o que sinaliza uma possível situação de insolvência ou excessiva alavancagem. Neste estudo, ficou demonstrado que não é interessante para empresas de Internet possuir resultados financeiros negativos, pois são empresas, em sua grande maioria, jovens e que necessitam demonstrar a sua capacidade de gerar lucros, que possam abrandar o razoável risco operacional envolvido neste novo setor da Economia.

A segunda variável proposta pelo modelo R\&D / EMP 1000 representa algo de bastante inovador nos estudos de análise de crédito. Esta variável não possui apenas como objetivo demonstrar um desempenho financeiro, embora seja, em sua essência, um índice monetário. O Modelo e-Score indica que empresas que apresentam valores mais altos para esta variável possuem uma probabilidade menor de falir. Esta seria uma conclusão óbvia, se o numerador da variável em exame não fosse um item de despesa de uma empresa. De fato, uma empresa que aumente qualquer item de despesa ou custo estará diminuindo o seu lucro no momento atual; entretanto, dependendo de qual item a empresa esteja majorando, poderão ocorrer benefícios futuros, que incrementarão os resultados da empresa.

É isto que o Modelo e-Score indica por meio desta variável. Empresas de Internet são reconhecidas como empresas que atribuem valor ao seu capital humano. Afinal, em muitos casos, são empresas dependentes da capacidade intelectual humana em desenvolver novas tecnologias e idéias. A alta significância apresentada pela variável R\&D / EMP 1000 no modelo indica que empresas de Internet bem sucedidas investem grandes somas em pesquisa e desenvolvimento, capacitando seus funcionários a atuar em tecnologias mais avançadas e modernas. Indica ainda, pelo fato de utilizar o número de 
empregados como parâmetro diferenciador entre empresas falidas e não-falidas, que este possa ser um identificador do tamanho de empresas de Internet, corroborando a idéia de que são empresas construídas e baseadas no capital humano.

A variável OCF / CL representa um ótimo indicador da eficiência operacional de uma empresa de Internet. Esta deve demonstrar ser capaz de pagar suas dívidas de curto prazo com seus próprios recursos, ou seja, deve apresentar um valor positivo para esta variável e, preferencialmente, maior que 1. Desta forma, a empresa sinaliza ao mercado que gera caixa suficiente para sobreviver no curto prazo, sem que seja necessária a intervenção de capital próprio ou de terceiros. Este conceito pode ser generalizado a empresas de vários setores da Economia, porém no Modelo e-Score demonstra possuir caráter discriminatório bastante forte para empresas de Internet.

O fato de que, mesmo nas empresas não-falidas, a média para esta variável se apresentou próxima ao zero demonstra uma característica já conhecida destas empresas: o seu curto tempo de operação. Em outros setores da Economia, empresas que estejam começando suas operações também demonstram uma incapacidade de gerar caixa suficiente para quitar suas dívidas. No setor de empresas de Internet, isto é mais preocupante ainda, pois as barreiras de entrada para novos entrantes, em geral, são muito pequenas. Isto faz com que uma empresa de Internet, ao iniciar suas operações, deva ser rápida em demonstrar aos seus acionistas e credores a sua capacidade de gerar caixa. Desta forma, o Modelo eScore indica que empresas de Internet, que não apresentam valores positivos e distantes do zero para a variável OCF / CL, possuem maior probabilidade de falência.

O Modelo e-Score demonstrou ser uma poderosa ferramenta na estimativa da probabilidade de falências de empresas de Internet. Portanto as instituições financeiras e os próprios acionistas poderiam estabelecer riscos de crédito diferenciados ou rentabilidades exigidas para empresas que apresentem um eScore acima de 0,5 , podendo também monitorá-las ou utilizar as variáveis propostas como covenants, o que corroboraria o fortalecimento desse setor da Economia, eliminando as empresas prejudiciais ao sistema que não produzem resultados satisfatórios.

\section{Artigo recebido em 03.11.2003. Aprovado em 23.01.2004.}




\section{ReferênCIAS Bibliográficas}

ALTMAN, E. I.

Financial ratios, discriminant analysis and the prediction of corporate bankruptcy. Journal of Finance, v. 23, set. 1968.

ALTMAN, E. I.;

BAYDIA, A.;

RIBEIRODIAS, L.

Assembling potential financial problems for firms in Brazil. Documento de Trabalho n. 8. PUCRJ. 1977.

BRAGANÇA, L.A.;

BRAGANÇA, S. L.

Rating, previsão de concordatas e falências no Brasil. Revista Brasileira de Mercado de Capitais. Rio de Janeiro: jan./mar. 1985.

\section{CABRERA, G.A. S.}

Um modelo neural para previsão de insolvência no sistema financeiro. Dissertação (Mestrado) - Departamento de Engenharia Elétrica da PUC-RJ. Rio de Janeiro, 1998.

\section{DEAKIN, E. B.}

A discriminant analysis of predictors of business failure. Journal of Accounting Research, mar./jun. 1972.

FICHMAN, L. H;

DA SILVA, J.F.

Construção de um modelo de predição de insolvência bancária baseada na tipologia de Porter. In: NESS, W.; VOLKEMA, R. (Orgs.). Estudos em Negócios, Estudos Coleção COPPEAD. Rio de Janeiro: Mauad Editores, 2001.
HEKANAHO, J. et al.

Analyzing bankruptcy with data with multiple methods. Turku: American Association for Artificial Intelligence, 1998.

HOSMER, D. W.;

LEMESHOW, S.

Applied Logistic Regression. 1. ed.

New York: John Wiley \& Sons, 1989.

JANOT, M. M.

Previsão de insolvência bancária no Brasil aplicação de diferentes modelos entre 1995 e 1998. Dissertação (Mestrado em Economia) - Departamento de Economia da PUCRJ. Rio de Janeiro, 1999.

KASZNAR, I. K.

Falências e concordatas de empresas. Modelos teóricos e estudos empíricos - 1978-1982. Dissertação (Mestrado em Economia) - Escola de Pós-Graduação em Economia da FGV. Rio de Janeiro, 1986.

MARQUES, J. N.

Previsão de insolvência de pequenas e médias empresas - uma aplicação da análise estatística multivariada. Dissertação (Mestrado em Administração) - Departamento de Administração da PUC-RJ. Rio de Janeiro, 1980. 
MARTINS, M. V.;

SAMANÉZ, J. O.

Análise discriminante e os bancos comerciais privados brasileiros: uma aplicação. Revista Brasileira de Mercado de Capitais. v. 13, n. 39. Rio de Janeiro: abr./ jun 1987.

MATIAS, A. B.

Contribuição às técnicas de análise financeira: um modelo de concessão de crédito. Trabalho de Formatura FEA/USP. São Paulo, 1978.

MATIAS, A. B.;

SIQUEIRA, J. O.

Risco bancário: modelo de previsão de insolvência de bancos no brasil. Revista de Administração da USP, v. 31, n. 2, abr./ jun. 1996.

\section{MINUSSI, J. A.}

Modelo preditivo de solvência utilizando regressão logística. Dissertação (Mestrado em Administração) - Departamento de Administração da PUC-RJ. Rio de Janeiro, 2001.

\section{MINUSSI, J. A.;}

DAMACENA, C.;

NESS JR., W. L.

Um modelo de previsão de solvência utilizando regressão logística. Revista deAdministração Contemporânea, v. 6, n. 3, p. 109-128, set./dez. 2002.
NUNES, V.M.

Um modelo de previsão de problemas financeiros graves em empresas. Dissertação (Mestrado em Administração) - Departamento de Administração da PUC-RJ. Rio de Janeiro, 1988.

\section{OHLSON, J.}

Financial Ratios and the Probabilistic Prediction of Bankruptcy. Journal of Accounting Research, v. 18, n.1, 1980.

SANTOS, S. C.

Um modelo de análise discriminante múltipla para previsão de inadimplência em empresa. Dissertação (Mestrado em Administração) - Departamento de Administração da PUC-RJ. Rio de Janeiro, 1996.

SINCICH, T.

Business Statistics by Example. 5. ed. New Jersey: Prentice-Hall, 1996.

\section{SINKEY, J.}

A Multivariate Statistical Analysis of the Characteristics of Problem Banks. Journal of Finance, p. 21-36, mar. 1975. 


\section{ANeXo 1: VariÁVEIS Estudadas}

1) CA/CL: Current Assets / Current Liabilities

3) PPE/AP: Total Net Property, Plant \& Equipment / (PP\&E + Goodwill + Intangible Assets $)$

5) (AP-LTL)/E: PP\&E + Goodwill + Intangible Assets - Long Term Liabilities) / Equity

7) NWC/REV: Net Working Capital / Total Revenue 8) AR/TA: Accounts Receivable / Total Assets

9) CASH/CA: Cash \& Short Term Investments / 10) CASH/TA: Cash \& Short Term Investments / Total Current Assets Assets

11)CASH/CL: Cash \& Short Term Investments / 12)EXP/EMP 1000: Total Selling, General and Current Liabilities

13)EXP/REV: Total Selling, General and Admin. Expenses / Total Revenue

15)INT/TL: Interest Expenses (Income) / Total Liabilities

17)EBT/TA: Earnings Before Tax / Total Assets

19)D/CA: Total Debt / Current Assets

21)D/E: Total Debt / Equity

23)AP/TA: (PP\&E + Goodwill + Intangible Assets $) /$ Total Assets

25) AP/REV: (PP\&E + Goodwill + Intangible Assets $)$ / Total Revenue

27)GP/REV: Gross Profit / Total Revenue

29) NI/TA: Net Income / Total Assets

31) NI/E: Net Income / Equity Admin. Expenses / $N^{o}$ de Empregados

14) INT/TA: Interest Expenses (Income) / Total Assets

16) INT/REV: Interest Expenses (Income) / Total Revenue

18) D/TA: Total Debt / Total Assets

20) D/CL: Total Debt / Current Liabilities

22) D/TL: Total Debt / Total Liabilities

33)(NI+INT)/TA: (Net Income + Interest Expenses (Income)) / Total Assets

35)(OI-DEPR)/GP: (Operating Income - Deprec., Depletion and Amortization) / Gross Profit

37)R\&D/EMP 1000: Research \& Development / $N^{\circ}$ de Empregados

39)CL/TA: Current Liabilities / Total Assets

41)E/TL: Equity / Total Liabilities

43)REV/EMP 1000: Total Revenue / $N^{o}$ de Empregados

45)RE/TA A2: Retained Earnings / Accumulated Deficit / Total Assets

47)EBITDA/TA: EBITDA / Total Assets

49)OCF/CL: Operating Cash Flow / Current Liabilities

51)FRCF/TA: Free Cash Flow / Total Assets

53)CL/(LTL+E): Current Liabilities / (Long Term Liabilities + Equity)

55)ICF/LTA: Investing Cash Flow / Long Term 56)CAPEX/REV: Capital Expenditures / Total Assets

57)FRCF/E: Free Cash Flow / Equity

59)EBITDA/OCF: EBITDA / Operating Cash Flow

61)OCF/SHREQ: Operating Cash Flow

Shareholders' Equity

63)EBITDA/EMP 1000: EBITDA / $N^{o}$ de Empregados

* As variáveis destacadas em tonalidades mais escuras são aquelas que foram consideradas significantes na discriminação das amostras de empresas falidas e não-falidas.

** As nomenclaturas contábeis estão no seu idioma original, pois foram utilizadas demonstrações de empresas norte-americanas e, desta forma, representam os princípios contábeis 